\title{
Combined optimal stopping and singular stochastic control
}

\author{
Ta Thi Kieu $A n^{1}$
}

Revised in May 2008

\begin{abstract}
In this paper a simple of combined singular stochastic control and optimal stopping in the jump-diffusion model is formulated and solved. We give sufficient conditions for the existence of an optimal strategy which has the same form as in continuous case given by Davis and Zervos [4] and also Karatzas et al. [8]. This result is applied to solve explicitly an example of such problem.
\end{abstract}

Key words: Jump-diffusions; Optimal harvest; Optimal stopping; Variation $\mathrm{Al}$ inequalities; Generalized Itô formula; Local time; Reflecting diffusions.

\section{Introduction}

The subject of the optimal singular control problem from a stochastic system described by a stochastic differential equation has been extensively studied by several authors, see e.g. [2], [3], [10], [11], [12] and [13] (Ch. 5). In our contribution, we consider a similar problem to that studied in [12], except that here optimal stopping is included in the control. Optimal stopping in control arises in target tracking problems where one has to decide when one has arrived sufficiently close to the target, see [4] and [8]. The problem of combined singular stochastic control and the optimal stopping problem also arise in the consumption/investment of financial economics for an investor who can decide when to exit from the market. In the paper by Karatzas

\footnotetext{
1 Centre of Mathematics for Applications (CMA), Department of Mathematics, University of Oslo, P.O. Box 1053 Blindern, N-0316 Oslo, Norway. Email: atkieu@math.uio.no
} 
and Wang [9], the stochastic optimization problem that combines features of both control and stopping is also considered by computing the upper- and lower-hedging prices of American contingent claims under constraints. Unlike these approaches, we study the case when the dynamics of the stochastic system therein has a jump component.

The paper is organized as follows: In the second section, we give and prove a verification theorem of the integro-variational inequality type suffice for optimal control and stopping. In Section 3, we apply the verification theorem to solve explicitly an example.

\section{Model}

We first recall that a Lévy process $\eta=\eta(t)=\left(\eta_{1}(t), \ldots, \eta_{n}(t)\right)$ on a complete probability $(\Omega, \mathcal{F}, \mathbf{P})$ is a process with stationary and independent increments and càdlàg path (i.e. right continuous paths with the left sided limits). The jump of $\eta_{i}$ at time $t$ is defined by

$$
\triangle \eta_{i}(t)=\eta_{i}(t)-\eta_{i}\left(t^{-}\right), \quad i=1, . ., n
$$

The jump measure $N_{i}$ of $\eta_{i}$ is defined by

$$
N_{i}((a, b], U):=\sum_{t \in(a, b]} 1_{U}\left(\triangle \eta_{i}(t)\right) ; \quad i=1, . . n,
$$

i.e. by the number of jumps of size $\triangle \eta_{i}(t) \in U$, for $t \in(a, b]$. Here $U$ is Borel set with $\bar{U} \subset \mathbb{R}_{0}:=\mathbb{R} \backslash\{0\}$. The differential form of this random measure is denoted by $N_{i}(d t, d z)$. The Lévy measure of $\eta_{i}$ is defined by

$$
\nu_{i}(U):=E\left[N_{i}((0,1], U)\right], \quad U \in \mathcal{B}\left(\mathbb{R}_{0}\right), \quad i=1, . . n
$$

and the compensated jump measure of $\eta_{i}$ is defined by

$$
\widetilde{N}_{i}(t, A)=N_{i}(t, A)-t \nu_{i}(A), \quad i=1, \ldots, n .
$$

In the sequel we assume that

$$
E\left[\eta_{i}^{2}(t)\right]<\infty, \quad t \geq 0, i=1, \ldots, n .
$$

The Lévy decomposition theorem states that if (1) holds, then there exists constants $a$ and $b$ such that

$$
\eta_{i}(t)=a t+b B_{i}(t)+\int_{0}^{t} \int_{\mathbb{R}_{0}} z \widetilde{N}_{i}(d s, d z), \quad t \geq 0, i=1, \ldots, n,
$$


where $B(t, \omega)=\left(B_{1}(t, \omega), \ldots, B_{n}(t, \omega)\right) ; t \geq 0$, is $n$-dimensional Brownian motion independent of $N$. In view of this, it is natural to study processes which has form

$$
\begin{aligned}
d X_{i}(t)= & b_{i}(t, X(t)) d t+\sum_{j=1}^{n} \sigma_{i j}(t, X(t)) d B_{j}(t) \\
& +\sum_{j=1}^{n} \int_{\mathbb{R}_{0}} \mu_{i j}\left(t^{-}, X\left(t^{-}\right), z_{j}\right) \widetilde{N}_{j}\left(d t, d z_{j}\right) \\
X_{i}(s)= & x_{i} \in \mathbb{R}, \quad 0 \leq s \leq t \leq T, i=1, \ldots, n
\end{aligned}
$$

where the coefficients $b: \mathbb{R} \times \mathbb{R}^{n} \rightarrow \mathbb{R}^{n}, \sigma: \mathbb{R} \times \mathbb{R}^{n} \rightarrow \mathbb{R}^{n \times n}$ and $\mu$ : $\mathbb{R} \times \mathbb{R}^{n} \times \mathbb{R}^{n} \rightarrow \mathbb{R}^{n \times n}$, are given $\mathcal{F}_{t^{-}}$adapted processes. Moreover, it is assumed that

$$
\begin{gathered}
E\left[\int _ { 0 } ^ { t } \left\{\left|b_{i}(s, X(s))\right|+\sigma_{i j}^{2}(s, X(s))\right.\right. \\
\left.\left.+\int_{\mathbb{R}_{0}} \mu_{i j}^{2}\left(s^{-}, X\left(s^{-}\right), z_{j}\right) \nu_{i}(d z)\right\} d s\right]<\infty \quad \text { a.s. } \quad \text { for all } t \geq 0 .
\end{gathered}
$$

For a detailed about such stochastic differential equation see e.g. [7], [14] and references given therein. We can consider equation (2) as the sizes or densities of population.

Let $S \subset \mathbb{R}^{n+1}$ (the solvency region) be a Borel set such that $S \subset \overline{S^{0}}$ where $S^{0}$ denotes the interior of $S, \overline{S^{0}}$ its closure. If we apply a control $\gamma(t, \omega)$ to the process $\left.X(t)=\left(X_{1}(t)\right), \ldots, X_{n}(t)\right)$ then the corresponding population vector $X^{(\gamma)}(t)=\left(X_{1}^{(\gamma)}(t), \ldots, X_{n}^{(\gamma)}(t)\right)^{T}$ becomes

$$
\begin{aligned}
d X^{(\gamma)}(t) & =b\left(t, X^{(\gamma)}(t)\right) d t+\sigma\left(t, X^{(\gamma)}(t)\right) d B(t) \\
& +\int_{\mathbb{R}_{0}^{n}} \mu\left(t^{-}, X^{(\gamma)}\left(t^{-}\right), z\right) \widetilde{N}(d t, d z)-d \gamma(t) ; \\
X^{(\gamma)}(s) & =x=\left(x_{1}, \ldots, x_{n}\right) \in \mathbb{R}^{n}, \quad s \leq t
\end{aligned}
$$

Here $\gamma(t)=\gamma(t, \omega)=\left(\gamma_{1}(t, \omega), \ldots, \gamma_{n}(t, \omega)\right) \in \mathbb{R}^{n}, t \geq s, \omega \in \Omega$ is nonnegative, non-decreasing, right-continuous and $\mathcal{F}_{t}$-adapted. Since $d \gamma(t)$ may be singular with respect to Lebesgue measure $d t$, we call $\gamma$ is our singular control. The component $\gamma_{i}(t, \omega)$ of $\gamma(t, \omega)$ can be considered as the total amount harvested from population number $i$ up to time $t$.

Let

$$
\tau_{S}=\tau_{S}(x, \omega)=\inf \left\{t>0 ;\left(t, X^{(\gamma)}(t)\right) \notin S\right\}
$$


be the time of extinction and let $\mathcal{T}$ denote the set of all stopping times $\tau \leq \tau_{S}$. And let the the prices/utilities per unit of population number $i$ accrued from harvesting at time $t$, be given by $n$ continuous, nonnegative functions

$$
\pi_{i}(t) ; \quad 1 \leq i \leq n
$$

Then the total expected discounted utility harvested from time $s$ to time $\tau \in \mathcal{T}$ is given by

$$
\begin{aligned}
J^{(\gamma, \tau)}(s, x)=E^{s, x} & {\left[\int_{s}^{\tau}\left\{f\left(t, X^{(\gamma)}(t)\right) d t+\pi(t) \cdot d \gamma(t)\right\}\right.} \\
& \left.+g\left(\tau, X^{(\gamma)}(\tau)\right) \cdot \chi_{\{\tau<\infty\}}\right]
\end{aligned}
$$

where $f: \mathbb{R} \times \mathbb{R}^{n} \rightarrow \mathbb{R}$ and $g: \mathbb{R} \times \mathbb{R}^{n} \rightarrow \mathbb{R}$ are continuous functions; $\pi=\left(\pi_{1}, \ldots, \pi_{n}\right) ; \pi \cdot d \gamma=\sum_{i=1}^{n} \pi_{i} d \gamma_{i}$; and $E^{s, x}$ denotes the expectation with respect to $P$ of the time-state process $\left(t, X^{(\gamma)}(t)\right), t \geq s$, when $X^{(\gamma)}(s)=x$.

We say that the control process $\gamma$ is admissible and write $\gamma \in \Gamma$ if (3) has a unique, strong solution $X^{(\gamma)}(t)$ and

$$
\begin{gathered}
E^{x}\left[\int_{0}^{\tau_{S}}\left|f\left(t, X^{(\gamma)}(t)\right)\right| d t+\mid g\left(\tau, X^{(\gamma)}(\tau) \mid \cdot \chi_{\{\tau<\infty\}}\right.\right. \\
\left.+\int_{0}^{\tau_{S}} \sum_{i=1}^{n}\left|\pi_{i}(t)\right| d \gamma_{i}(t)\right]<\infty .
\end{gathered}
$$

The optimal stopping and singular stochastic control problem is to find the value function $\Phi(s, x)$ and an optimal strategy $\left(\gamma^{*}, \tau^{*}\right) \in \Gamma \times \mathcal{T}$ (if exists) such that

$$
\Phi(s, x)=\sup _{\gamma \in \Gamma, \tau \in \mathcal{T}} J^{(\gamma, \tau)}(s, x)=J^{\left(\gamma^{*}, \tau^{*}\right)}(s, x)
$$

If we do not apply any harvesting, then the generator of corresponding time-state population process $(t, X(t))$, with $X(t)$ given by (2), coincides on $C_{0}^{2}\left(\mathbb{R}^{n+1}\right)$ with partial differential operator $L$ given by

$$
\begin{aligned}
L g(s, x) & =\frac{\partial g}{\partial s}(s, x)+\sum_{i=1}^{n} b_{i}(s, x) \frac{\partial g}{\partial x_{i}}(s, x) \\
+ & \frac{1}{2} \sum_{i, j=1}^{n}\left(\sigma \sigma^{T}\right)_{i j}(s, x) \frac{\partial^{2} g}{\partial x_{i} \partial x_{j}}(s, x) \\
+ & \sum_{j=1}^{n} \int_{\mathbb{R}}\left\{g\left(s, x+\mu_{j}\left(s, x, z_{j}\right)\right)-g(s, x)\right. \\
& \left.-\nabla g(s, x) \mu_{j}\left(s, x, z_{j}\right)\right\} \nu_{j}\left(d z_{j}\right)
\end{aligned}
$$


Note the difference between the jumps of $X^{(\gamma)}(t)$ caused by the jump of $N(d t, d z)$, denote by $\triangle_{N} X^{(\gamma)}(t)$, and the jump caused by the harvesting, denoted by $\triangle_{\gamma} X^{(\gamma)}(t)$. Thus

$$
\triangle_{N} X^{(\gamma)}(t)=\int_{\mathbb{R}^{n}} \mu\left(t^{-}, X^{(\gamma)}\left(t^{-}\right), z\right) \tilde{N}(d t, d z),
$$

while

$$
\triangle_{\gamma} X^{(\gamma)}(t)=-\triangle \gamma(t)
$$

We will in the following let $t_{1}, t_{2}, \ldots$ denote the jumping times of a given strategy $\gamma \in \Gamma$. The jump of $\gamma(t)$ at $t=t_{k}$ are

$$
\triangle \gamma\left(t_{k}\right)=\gamma\left(t_{k}\right)-\gamma\left(t_{k}^{-}\right)
$$

And we let

$$
\gamma^{(c)}(t):=\gamma(t)-\sum_{s \leq t_{k} \leq t} \Delta \gamma\left(t_{k}\right)
$$

be the continuous part of $\gamma(t)$.

If $\phi$ is a continuous real function on $S$ and we let

$$
\triangle_{\gamma} \phi\left(t_{k}, X^{(\gamma)}\left(t_{k}\right)\right)=\phi\left(t_{k}, X^{(\gamma)}\left(t_{k}\right)\right)-\phi\left(t_{k}^{-}, X^{(\gamma)}\left(t_{k}^{-}\right)+\triangle_{N} X^{(\gamma)}\left(t_{k}\right)\right)
$$

denote the jump in the value of $\phi\left(t_{k}, X^{(\gamma)}\left(t_{k}\right)\right)$ caused by the jump of $\gamma$ at $t=t_{k}$. We emphasize that the possible jumps in $X^{(\gamma)}\left(t_{k}\right)$ coming from $\widetilde{N}$ are not included in $\triangle_{\gamma} \phi\left(t_{k}, X^{(\gamma)}\left(t_{k}\right)\right)$.

We now formulate a sufficient condition for a given function $\phi(s, x)$ to be the value function $\Phi(s, x)$ of $(7)$ and for a given pair $\left(\gamma^{*}, \tau^{*}\right)$ to be optimal.

\section{Theorem 2.1. (A verification theorem)}

a) Suppose there exists a function $\phi \in C^{1}(S) \cap C(\bar{S})$ such that

(i) $\phi(t, x) \geq g(t, x)$ for all $(s, x) \in S$

(ii) $\frac{\partial \phi}{\partial x_{i}}(t, x) \geq \pi_{i}(t)$ for all $(s, x) \in S, i=1, \ldots, n$

(iii) $L \phi(t, x)+f(t, x) \leq 0$ for all $(s, x) \in S$

(iv) $\left(\tau_{S}, X^{(\gamma)}\left(\tau_{S}\right)\right) \in \partial S$ a.s. on $\tau_{S}<\infty$ and $\lim _{t \rightarrow \tau_{S}^{-}} \phi\left(t, X^{(\gamma)}(t)\right)=g\left(\tau_{S}, X^{(\gamma)}\left(\tau_{S}\right)\right) \chi_{\left\{\tau_{S}<\infty\right\}}$ a.s for all $\gamma \in \Gamma$

Then

$$
\phi(s, x) \geq \Phi(s, x) \quad \text { for all }(s, x) \in S
$$


b) Define the continuation region $D$ by

$$
D:=\{(t, x) \in S ; \phi(t, x)>g(t, x)\}
$$

In additional (i)-(iv) above, we assume that there exists a control $(\hat{\gamma}, \hat{\tau}) \in \Gamma \times \mathcal{T}$ such that

(v) $L \phi(t, x)+f(t, x)=0$ for all $(t, x) \in D$

(vi) $\left(\frac{\partial \phi}{\partial x_{i}}\left(t, X^{(\hat{\gamma})}(t)\right)-\pi_{i}(t)\right) d \hat{\gamma}_{i}^{(c)}(t)=0$ for all $t ; i=1, \ldots, n$, where $\hat{\gamma}^{(c)}$ is continuous part of $\hat{\gamma}(t)$

(vii) $\triangle_{\hat{\gamma}} \phi\left(t_{k}, X^{(\hat{\gamma})}\left(t_{k}\right)\right)=-\pi\left(t_{k}\right) \cdot \triangle \hat{\gamma}\left(t_{k}\right)$ for all jumping time $t_{k}$ of $\hat{\gamma}(t)$

(viii) $\lim _{R \rightarrow \infty} E^{y}\left[\phi\left(T_{R}, X^{(\hat{\gamma})}\left(T_{R}\right)\right)\right]=E^{y}\left[g\left(\tau_{S}, X^{(\hat{\gamma})}\left(T_{R}\right)\right) \cdot \chi_{\left\{\tau_{S}<\infty\right\}}\right]$ where $T_{R}=\min \left(\tau_{S}, R\right)$, for $R<\infty$

(ix) $\tau_{D}:=\inf \left\{t>0 ;\left(t, X^{(\hat{\gamma})}(t)\right) \notin D\right\}<\infty$ a.s. for all $x \in S$

(x) $\left\{\phi\left(s, X^{(\gamma)}(\tau)\right) ; \tau \in \mathcal{T}, \tau \leq \tau_{D}\right\}$ is uniformly integrable, for all $x \in S,(\gamma, \tau) \in \Gamma \times \mathcal{T}$

Then

$$
\phi(s, x)=\Phi(s, x) \quad \text { for all }(s, x) \in S
$$

and

$$
\left(\gamma^{*}, \tau^{*}\right)=\left(\hat{\gamma}, \tau_{D}\right)
$$

is a pair of optimal strategy.

Proof. a) Choose $\gamma \in \Gamma$ and assume that $\phi \in C^{2}\left(\mathbb{R}^{n+1}\right)$ satisfies the conditions of a). Let $\tau \leq \tau_{S}$ be a stopping time, $m \in \mathbb{N}$. Then by Itô's formula for semimartingale (see e.g. Protter (1990), Th. II.7.33, [14]) we have

$$
\begin{aligned}
& E^{s, x}\left[\phi\left(\tau \wedge m, X^{(\gamma)}(\tau \wedge m)\right)\right]=E^{s, x}\left[\phi\left(s, X^{(\gamma)}(s)\right)\right] \\
& +E^{s, x}\left[\int_{s}^{\tau \wedge m} L \phi\left(t, X^{(\gamma)}(t)\right) d t-\int_{s}^{\tau \wedge m} \sum_{i=1}^{n} \frac{\partial \phi}{\partial x_{i}}\left(t, X^{(\gamma)}(t)\right) d \gamma_{i}(t)\right. \\
& +\sum_{s<t_{k} \leq \tau \wedge m}\left\{\triangle_{\gamma} \phi\left(t_{k}, X^{(\gamma)}\left(t_{k}\right)\right)\right. \\
& \left.\left.\quad-\sum_{i=1}^{n} \frac{\partial \phi}{\partial x_{i}}\left(t_{k}, X^{(\gamma)}\left(t_{k}\right)\right) \triangle_{\gamma} X_{i}^{(\gamma)}\left(t_{k}\right)\right\}\right]
\end{aligned}
$$


where $t_{k}$ denotes the times of jumps for $\gamma(t)$ and

$$
\triangle_{\gamma} X_{i}^{(\gamma)}\left(t_{k}\right)=X_{i}^{(\gamma)}\left(t_{k}\right)-\left(X_{i}^{(\gamma)}\left(t_{k}^{-}\right)+\triangle_{N} X_{i}^{\gamma}\left(t_{k}\right)\right)
$$

Then (11) can be written

$$
\begin{aligned}
E^{s, x}[\phi(\tau \wedge m, & \left.\left.X^{(\gamma)}(\tau \wedge m)\right)\right]=\phi(s, x)+E^{s, x}\left[\int_{s}^{\tau \wedge m} L \phi\left(t, X^{(\gamma)}(t)\right) d t\right] \\
- & E^{s, x}\left[\int_{s}^{\tau \wedge m} \sum_{i=1}^{n} \frac{\partial \phi}{\partial x_{i}}\left(t, X^{(\gamma)}(t)\right) d \gamma_{i}^{(c)}(t)\right] \\
+ & E^{s, x}\left[\sum_{s<t_{k} \leq \tau \wedge m} \triangle_{\gamma} \phi\left(t_{k}, X^{(\gamma)}\left(t_{k}\right)\right)\right]
\end{aligned}
$$

Therefore

$$
\begin{aligned}
& \phi(s, x)=E^{s, x}\left[\phi\left(\tau \wedge m, X^{(\gamma)}(\tau \wedge m)\right)-\int_{s}^{\tau \wedge m} L \phi\left(t, X^{(\gamma)}(t)\right) d t\right. \\
& \left.+\int_{s}^{\tau \wedge m} \sum_{i=1}^{n} \frac{\partial \phi}{\partial x_{i}}\left(t, X^{(\gamma)}(t)\right) d \gamma_{i}^{(c)}(t)-\sum_{s<t_{k} \leq \tau \wedge m} \triangle_{\gamma} \phi\left(t_{k}, X^{(\gamma)}\left(t_{k}\right)\right)\right]
\end{aligned}
$$

By the mean value theorem we have

$$
\begin{aligned}
-\triangle_{\gamma} \phi\left(t_{k}, X^{(\gamma)}\left(t_{k}\right)\right) & =\sum_{i=1}^{n} \frac{\partial \phi}{\partial x_{i}}\left(t_{k}, \widehat{X}^{(k)}\left(t_{k}\right)\right) \triangle_{\gamma} X_{i}^{(\gamma)}\left(t_{k}\right) \\
& =\sum_{i=1}^{n} \frac{\partial \phi}{\partial x_{i}}\left(t_{k}, \widehat{X}^{(k)}\right) \triangle \gamma_{i}\left(t_{k}\right)
\end{aligned}
$$

where $\widehat{X}^{(k)}$ is some points on the straight line between $X^{(\gamma)}\left(t_{k}\right)$ and $X^{(\gamma)}\left(t_{k}^{-}\right)+$ $\triangle_{N} X^{(\gamma)}\left(t_{k}\right)$. Therefore, invoking conditions (i) and (ii) hold, then by (13) and (14) we have 


$$
\begin{aligned}
\phi(s, x) \geq E^{s, x}\left[\phi\left(\tau \wedge m, X^{(\gamma)}(\tau \wedge m)\right)+\int_{s}^{\tau \wedge m} f\left(t, X^{\gamma}(t)\right) d t\right] \\
+E^{s, x}\left[\sum_{i=1}^{n}\left\{\int_{s}^{\tau \wedge m} \pi_{i}(t) d \gamma_{i}^{(c)}(t)+\sum_{s<t_{k} \leq \tau \wedge m} \pi_{i}\left(t_{k}\right) \triangle \gamma_{i}\left(t_{k}\right)\right\}\right] \\
=E^{s, x}\left[\phi\left(\tau \wedge m, X^{(\gamma)}(\tau \wedge m)\right)+\int_{s}^{\tau \wedge m} f\left(t, X^{\gamma}(t)\right) d t\right] \\
+E^{s, x}\left[\int_{s}^{\tau \wedge m} \pi(t) \cdot d \gamma^{(c)}(t)+\sum_{s<t_{k} \leq \tau \wedge m} \pi\left(t_{k}\right) \cdot \triangle \gamma\left(t_{k}\right)\right] \\
=E^{s, x}\left[\int_{s}^{\tau \wedge m}\left\{f\left(t, X^{\gamma}(t)\right) d t+\pi(t) \cdot d \gamma(t)\right\}\right. \\
\left.\quad+\phi\left(\tau \wedge m, X^{(\gamma)}(\tau \wedge m)\right)\right]
\end{aligned}
$$

By letting now $m \rightarrow \infty$, we obtain by monotone convergence theorem that for any admissible control $(\gamma, \tau) \in \Gamma \times \mathcal{T}$ and for all $x \in \mathbb{R}^{n}$

$$
\begin{aligned}
\phi(s, x) \geq E^{s, x}\left[\int _ { s } ^ { \tau } \left\{f\left(t, X^{\gamma}(t)\right) d t+\pi(t) \cdot d \gamma(t)\right.\right. \\
\left.\quad+g\left(\tau, X^{(\gamma)}(\tau)\right) \chi_{\{\tau<\infty\}}\right] \\
=J^{(\tau, \gamma)}(s, x)
\end{aligned}
$$

Since (16) is valid for any admissible strategy, a) is proved.

b) Now consider $D$ as above and with conditions (v)-(x). Then apply the argument above to strategy $\hat{\gamma} \in \Gamma$ we now have equality

$$
\begin{aligned}
\phi(s, x)= & E^{s, x}\left[\phi\left(\tau \wedge m, X^{(\hat{\gamma})}(\tau \wedge m)\right)+\int_{s}^{\tau \wedge m} f\left(t, X^{(\hat{\gamma})}(t)\right) d t\right] \\
+ & E^{s, x}\left[\int_{s}^{\tau \wedge m} \pi(t) \cdot d \hat{\gamma}^{(c)}(t)+\sum_{s<t_{k} \leq \tau \wedge m} \pi\left(t_{k}\right) \cdot \triangle \hat{\gamma}\left(t_{k}\right)\right] \\
= & E^{s, x}\left[\phi\left(\tau \wedge m, X^{(\hat{\gamma})}(\tau \wedge m)\right)+\int_{s}^{\tau \wedge m} f\left(t, X^{(\hat{\gamma})}(t)\right) d t\right] \\
& \quad+E^{s, x}\left[\int_{s}^{\tau \wedge m} \pi(t) \cdot d \hat{\gamma}(t)\right] \\
\rightarrow & \left.J^{\left(\tau^{*}, \hat{\gamma}\right.}\right)(s, x) \quad \text { as } m \rightarrow \infty .
\end{aligned}
$$


Hence $\phi(s, x)=J^{\left(\tau^{*}, \gamma^{*}\right)}(s, x) \leq \Phi(s, x)$ with $\gamma^{*}=\hat{\gamma}$. This together with (9) proves the requirements (10) and then $\left(\gamma^{*}, \tau^{*}\right)$ is an optimal strategy for problem (7).

This completes the proof of the verification theorem.

\section{Example.}

In this section we apply Theorem 1 to an example in target tracking problem where one has to decide when one is "sufficiently close" to the target.

Suppose a controlled stochastic system of jump type $X(t)$ at time $t$ is modeled by geometric Lévy process, i.e.

$$
\begin{aligned}
d X^{(\gamma)}(t) & =X^{(\gamma)}\left(t^{-}\right)\left[\mu d t+\sigma d B(t)+\int_{\mathbb{R}} z \widetilde{N}(d t, d z)\right]-d \gamma(t), \\
X^{(\gamma)}(s) & =x>0
\end{aligned}
$$

where $\mu, \sigma>0$ are constants, and $\gamma(t)$ is right-continuous and increasing adapted process giving the amount harvested from time 0 up to time $t$. We assume that all jumps are positive, i.e.

$$
z>0 \quad \text { a.s. } \nu \text {. }
$$

The objective of the control is to keep the process $X(t)$ as close to the the origin as possible up to a stopping time $\tau$, and it is measured by the functional

$$
\begin{aligned}
J^{(\gamma, \tau)}(s, x) & =E^{s, x}\left[\int_{0}^{\tau} e^{-\rho(s+t)}\{\alpha d t+d \gamma(t)\}\right. \\
& \left.+e^{-\rho(s+\tau)} \lambda\left(X^{(\gamma)}(\tau)\right)^{2} \cdot \chi_{\{\tau<\infty\}}\right]
\end{aligned}
$$

here $\alpha, \rho, \lambda>0$ are given constants. The problem is that we try to minimize the expected discounted total cost function (19), i.e. we want to compute the minimal such expected cost

$$
\Phi(s, x)=\inf _{(\gamma, \tau) \in \mathcal{A} \times \mathcal{T}} J^{(\gamma, \tau)}(s, x)=J^{\left(\gamma^{*}, \tau^{*}\right)}(s, x)
$$

where $\mathcal{A}$ and $\mathcal{T}$ are the class of admissible controls $\gamma$ and the class of all stopping times $\tau$, respectively.

This is problem of the type described above, except that it is a minimum problem rather than a maximum problem. Theorem 1 still applies, with the corresponding changes. 
We define the solvency region by

$$
S=\mathbb{R} \times(0, \infty)
$$

If there is no control strategy, the generator of time-space process $(t, X(t))$ is given by

$$
\begin{aligned}
L \phi(s, x) & =\frac{\partial \phi}{\partial s}(s, x)+x \mu \frac{\partial \phi}{\partial x}(s, x)+\frac{1}{2} x^{2} \sigma^{2} \frac{\partial^{2} \phi}{\partial x^{2}}(s, x) \\
& +\int_{\mathbb{R}}\left\{\phi(s, x+x z)-\phi(s, x)-x z \frac{\partial \phi}{\partial x}(s, x)\right\} \nu(d z)
\end{aligned}
$$

We conjecture the function $\phi$ of form

$$
\phi(s, x)=e^{-\rho s} x^{r}, \quad \text { for some constant } r \in \mathbb{R} .
$$

Substituting this form into the equation (21), we come to equation

$$
\begin{aligned}
L \phi(s, x) & =e^{-\rho s} x^{r}\left[-\rho+\mu r+\frac{1}{2} \sigma^{2} r(r-1)\right. \\
& \left.+\int_{\mathbb{R}}\left\{(1+z)^{r}-1-z r\right\} \nu(d z)\right]
\end{aligned}
$$

Since the stopping cost $\lambda\left(X^{(\gamma)}(\tau)\right)^{2}$ is minimum at $x=0$, it is natural to predict that the stopping set is a neighborhood of zero. One strategy is to "do nothing" if process is inside $(a, \infty)$ and to stop as soon as the process hits the set $[0, a]$. Hence, we conjecture that the continuation region $D$ has the following form

$$
D=\{(t, x): x>a\}, \quad \text { for some a } \geq 0 .
$$

In the continuation region, the principle of optimal control requires $L \phi(s, x)+f(x)=0$, or

$$
\begin{gathered}
e^{-\rho s} x^{r}\left[-\rho+\mu r+\frac{1}{2} \sigma^{2} r(r-1)\right. \\
\left.+\int_{\mathbb{R}}\left\{(1+z)^{r}-1-z r\right\} \nu(d z)\right]+e^{-\rho s} \alpha=0,
\end{gathered}
$$

Putting

$$
h(r):=-\rho+\mu r+\frac{1}{2} \sigma^{2} r(r-1)+\int_{\mathbb{R}}\left\{(1+z)^{r}-1-z r\right\} \nu(d z)
$$


Note that

$$
h(1)=\mu-\rho \quad \text { and } \quad \lim _{r \rightarrow \infty} h(r)=0
$$

Therefore, if we assume that

$$
\mu<\rho
$$

then we get that there exists $r_{1} \in\left(1, \frac{\rho}{\mu}\right)$ such that

$$
h\left(r_{1}\right)=0
$$

Hence equation (23) admits a general solution of the following form

$$
\phi(s, x)=e^{-\rho s} C x^{r_{1}}+\frac{\alpha}{\rho}
$$

where $C$ is arbitrary constants. The cost function of this strategy is

$$
\phi(s, x)=\left\{\begin{array}{lcc}
e^{-\rho s} \lambda x^{2} & \text { if } & 0 \leq x \leq a \\
e^{-\rho s}\left(C x^{r_{1}}+\frac{\alpha}{\rho}\right) & \text { if } & a<x
\end{array}\right.
$$

We guess that the value function $\phi$ is $C^{1}$ at $x=a$ and this gives the following "hight contact"- conditions:

$$
C a^{r_{1}}+\frac{\alpha}{\rho}=\lambda a^{2} \quad(\text { continuity at } x=a)
$$

and

$$
r_{1} C a^{r_{1}-1}=2 \lambda a \quad(\text { differentiability at } x=a)
$$

The solution to these equations is

$$
C=\frac{2 \lambda}{r_{1}} a^{-r_{1}+2}, \quad a^{2}=\frac{-\alpha r_{1}}{\lambda \rho\left(2-r_{1}\right)}
$$

It is easy to check that the function $\phi$ defined by (27) and (28) satisfies the condition (ii) of the Theorem 1 if and only if $a \leq \frac{1}{2 \lambda}$, i.e.

$$
\alpha \geq \frac{\rho\left(r_{1}-2\right)}{4 \lambda r_{1}} .
$$

Because of this condition, we have to look for further possible strategy. Another possible strategy is to introduce two barrier points $0<a<b$. This strategy can be explained as follows: if the process is inside the set $(b, \infty)$, we will move it immediately to the reflecting barrier $b$; we will do nothing if the process is in the set $(a, b]$ and stop it if it is in $[0, a]$. 
If this strategy is optimal for some $a, b$, then we conjecture that the cost function $\phi(s, x)=J^{\left(\gamma^{*}, \tau^{*}\right)}$ will be $C^{1}$ at $a$ and $b$. In the set $(b, \infty), \phi$ will satisfy

$$
L \phi(s, x)+f(x)=0, \quad \text { for } x \in(b, \infty) .
$$

The general solution of this equation is given by (26). Outside the region $(b, \infty)$, the value function $\phi$ is described by

$$
\phi(s, x)=e^{-\rho s} \lambda x^{2}, \quad \text { for all } x \in[0, a],
$$

and

$$
\phi(s, x)=e^{-\rho s}\left(x-a+\lambda a^{2}\right), \quad \text { for all } x \in(a, b]
$$

We now summarize the cost function for this strategy as following,

$$
\phi(s, x)=\left\{\begin{array}{lcc}
e^{-\rho s} \lambda x^{2} & \text { if } & 0 \leq x \leq a \\
e^{-\rho s}\left(x-a+\lambda a^{2}\right) & \text { if } & a<x \leq b \\
e^{-\rho s}\left(C x^{r_{1}}+\frac{\alpha}{\rho}\right) & \text { if } & b<x
\end{array}\right.
$$

Again, assuming $C^{1}$ fits at both points $a$ and $b$, we obtain a following system of three equations for three unknown constants $C, a, b$ :

$$
\begin{aligned}
2 \lambda a & =1 \\
C b^{r_{1}}+\frac{\alpha}{\rho} & =b-a+\lambda a^{2} \\
r_{1} C b^{r_{1}-1} & =1
\end{aligned}
$$

The solution to this system is

$$
a=\frac{1}{2 \lambda}, \quad b=\frac{-r_{1}}{1-r_{1}} \frac{\rho+4 \alpha \lambda}{4 \lambda \rho}, \quad C=\frac{1}{r_{1}} b^{-r_{1}+1}
$$

From (33) we conclude that $a<b$ if and only if

$$
\alpha<\frac{\rho\left(r_{1}-2\right)}{4 \lambda r_{1}} .
$$

which is complement of condition (18).

It remains to verify that with these values of $a, b$, and $C$ the function $\phi(s, x)$ given by (27) and (32) satisfy all the conditions (i)-(x) of Theorem 1. 
To this end, first note that the condition (v) holds by construction of $\phi$. Moreover, $\phi=g$ outside $D=\{(s, x) ; x>a\}$. Therefore, to verify (i) we only need to prove that:

$$
\phi \leq g \quad \text { on } D
$$

We first prove (35) holds for the case $\alpha \geq \frac{\rho\left(r_{1}-2\right)}{4 \lambda r_{1}}$, i.e. we prove

$$
C x^{r_{1}}+\frac{\alpha}{\rho} \leq \lambda x^{2} \quad \text { for } x>a,
$$

Define

$$
k(x)=C x^{r_{1}}-\lambda x^{2}+\frac{\alpha}{\rho}
$$

By our chosen values of $a$ and $C$ as in (28), we have $k(a)=k^{\prime}(a)=0$. Moreover,

$$
k^{\prime \prime}(x)=C r_{1}\left(r_{1}-1\right) x^{r_{1}-2}-2 \lambda<0 \quad \text { for } x>a
$$

Therefore $k(x)<0$ for $x>a$ and (36) holds.

Secondly, we prove that if $\alpha<\frac{\rho\left(r_{1}-2\right)}{4 \lambda r_{1}}$ then

$$
x-a+\lambda a^{2} \leq \lambda x^{2}, \quad \text { for } a<x \leq b .
$$

Define $F(x)=\lambda x^{2}-x+a-\lambda a^{2}$. With the values of $a$ and $C$ in (33) we have $F(a)=0$ and $F^{\prime}(x)>0$ for $x>a$. So inequality (37) holds for all $a<x \leq b$ and hence (i) is proved.

(ii) Condition (ii) is checked in the same way as we do in (i).

(iii) Outside $D$ we have

$$
\phi(s, x)=e^{-\rho s} \lambda x^{2} \quad \text { for } a \leq x
$$

and

$$
\phi(s, x)=e^{-\rho s}\left(x-a+\lambda a^{2}\right) \quad \text { for } a<x \leq b
$$

Therefore $L \phi(s, x)+f(s, x) \geq 0$ gets the following forms:

$$
\left(-\rho+2 \mu+\sigma^{2}+\int_{\mathbb{R}} z^{2} \nu(d z)\right) \lambda x^{2}+\alpha \geq 0 \quad \text { for } x \leq a
$$

which is equivalent to

$$
-\rho+2 \mu+\sigma^{2}+\int_{\mathbb{R}} z^{2} \nu(d z) \geq 0 ;
$$


and

$$
x(\mu-\rho)+\frac{\rho+4 \lambda \alpha}{4 \lambda} \geq 0 \quad \text { for } a<x \leq b
$$

Since $\mu<\rho$, we get from (40) that

$$
\begin{aligned}
x(\mu-\rho) & +\frac{\rho+4 \lambda \alpha}{4 \lambda} \geq 0, \quad \text { for } a<x \leq b \\
& \mathbb{1} \\
b(\mu-\rho) & +\frac{\rho+4 \lambda \alpha}{4 \lambda} \geq 0 \\
& \mathbb{1} \\
b & \geq-\frac{\rho+4 \lambda \alpha}{4 \lambda(\mu-\rho)} \\
& \mathbb{1} \\
r_{1} & \geq \frac{\rho}{\mu}
\end{aligned}
$$

(iv) holds since we assume that $z>0$ a.s. $\nu$.

(vi)-(viii): These conditions claim the existence of an increasing process $\hat{\gamma}$, that is local time at $b$ of the downward reflected process $X^{(\gamma)}(t)$. (See [13], Th. 5.4.)

(ix) Suppose $Z(t)$ be the càdlàg adapted solution of the stochastic equation

$$
\begin{aligned}
d Z(t) & =Z\left(t^{-}\right)\left[\mu d t+\sigma d B(t)+\int_{\mathbb{R}} z \widetilde{N}(d t, d z)\right] \\
Z(0) & =x>0
\end{aligned}
$$

Let $\mathcal{C}$ be the set of càdlàg functions $f:[0, \infty) \rightarrow \mathbb{R}$. Define the map $G: \mathcal{C} \rightarrow \mathcal{C}$ by

$$
G(f)(t)=f(t)+\sup _{s \leq s \leq t} f(s)
$$

and define the map $\Lambda: \mathcal{C} \rightarrow \mathcal{C}$ by

$$
\Lambda(f)(t)=G(f)(t)-f(t)=\sup _{s \leq s \leq t} f(s)
$$

Then $X^{(\gamma)}(t):=G(Z)(t)$ and $\gamma(t):=\Lambda(Z)(t)$ is solution of equation (17). To check $\tau_{D}<\infty$ a.s. we consider the solution $Z(t)$ of equation (42) given by

$$
Z(t)=x \exp \left\{\left(\mu-\frac{1}{2} \sigma^{2}-\int_{\mathbb{R}} z \nu(d z)\right) t+\int_{0}^{t} \int_{\mathbb{R}} \ln (1+z) N(d t, d z)+\sigma B(t)\right\}
$$


Since $z>0$ a.s. $\nu$ and apply the law of iterated logarithm we see that if

$$
\mu-\frac{1}{2} \sigma^{2}-\int_{\mathbb{R}} z \nu(d z)>0
$$

then

$$
\lim _{t \rightarrow \infty} Z(t)=\infty \quad \text { a.s }
$$

Hence $\lim _{t \rightarrow \infty} X^{(\gamma)}(t)=\infty$ a.s. and particular $\tau_{D}<\infty$.

We now summarize the results of the problem (20) in the following results:

Theorem 3.1. Let $X^{(\gamma)}(t)$ be given by (17). Denote by $r_{1}$ a root of the equation (23). Suppose that (25), (39), (41) and (43) hold. Then

a) If

$$
\alpha \geq \frac{\rho\left(r_{1}-2\right)}{4 \lambda r_{1}},
$$

the value function $\phi(s, x)$ is given explicitly as

$$
\phi(s, x)=\left\{\begin{array}{lcc}
e^{-\rho s} \lambda x^{2} & \text { if } & 0 \leq x \leq a \\
e^{-\rho s}\left(C x^{r_{1}}+\frac{\alpha}{\rho}\right) & \text { if } & a<x
\end{array}\right.
$$

where $C$ and $a>0$ are given by (28).

b) If

$$
\alpha<\frac{\rho\left(r_{1}-2\right)}{4 \lambda r_{1}}
$$

The value function $\phi(s, x)$ is given explicitly as

$$
\phi(s, x)=\left\{\begin{array}{lcc}
e^{-\rho s} \lambda x^{2} & \text { if } 0 \leq x \leq a \\
e^{-\rho s}\left(x-a+\lambda a^{2}\right) & \text { if } & a<x \leq b \\
e^{-\rho s}\left(C x^{r_{1}}+\frac{\alpha}{\rho}\right) & \text { if } & b<x
\end{array}\right.
$$

where $a, b$ and $C$ are given by (33). The corresponding optimal policy is following:

- Stop immediately if $0 \leq x \leq a: \tau^{*}=0$, 
- Do nothing if $a<x \leq b$,

- Apply the harvesting equal to the local time of the reflected process $X^{(\gamma)}(t)$ at $b$.

Acknowledgments. The author wishes to express her thanks to Prof. Bernt Øksendal for valuable comments and discussions.

\section{References}

[1] Alvarez, L. H. R., Singular stochastic control in the presence of a statedependent yield structure. Stochastic Processes and Their Application 2000, 86, 323-343.

[2] Alvarez, L. H. R. and Shepp, L. A., Optimal harvesting of stochastically fluctuating populations. J. Math. Biol. 1998, 37, 155-177.

[3] Alvarez, L. H. R., Lungu, E. and Øksendal, B., Optimal multi-dimensional stochastic harvesting with density-dependent prices. Bernoulli Journal 2001, 7 (3), 527-539.

[4] Davis, M. H. A. and Zervos, M., A problem of singular stochastic control with discretionary stopping. Annals of Appl. Prob. 1994, 4 (1), 226-240.

[5] Framstad, N. C., Øksendal, B. and Sulem, A., Optimal Consumption and Portfolio in a Jump Diffusion Market. in: A. Shiryaev and A. Sulem, eds., Workshop on Mathematical Finance, (INRIA, Paris), (1998).

[6] Framstad, N. C., Øksendal, B. and Sulem. A., Optimal consumption and portfolio in a jump diffusion market with proportional transaction costs. J. Math. Econ. 2001, 191, 1-25.

[7] Jacod, J. and Shiryaev, A., Limit Theorems for Stochastic Processes. Springer-Verlag, New York, 1995.

[8] Karatzas, I., Ocone, D, Wang, H. and Zervos, M., Finite-fuel singular control with discretionary stopping. Stochastics and Stochastics Reports $2000,71,1-50$.

[9] Karatzas, I. and Wang, H., A barrier option of American type. Applied Mathematics and Optimization. 
[10] Lungu, E. and Øksendal, B., Optimal harvesting from a population in a stochastic crowded environment. Math. Biosci. 1997, 145, 47-75.

[11] Lungu, E. and Øksendal, B., Optimal harvesting from interacting population in a stochastic environment. Bernoulli Journal2001, 7, 527-539.

[12] Makasu, M., On a problem of optimal harvesting from a stochastic system with a jump component. Stochastics and Stochastics Reports 2002, 73 (3-4), 333-347.

[13] Øksendal, B. and Sulem, A., Applied Stochastic Control of Jump Diffusions. Second Edition. Springer, 2007.

[14] Protter, P., Stochastic Integration and Differential Equations. Application of mathematics (New York) 21, Springer-Verlag, Berlin, Heidelberg, 1995, A new Approach. 